\title{
Tratamento endodôntico de dente com necrose pulpar e lesão periapical com instrumentos Reciproc Blue e XP-Endo Finisher: relato de caso clínico
}

\author{
Endodontic treatment in tooth with pulp necrosis and periapical lesion with Reciproc Blue and XP-
} Endo Finisher instruments: case report

Tratamiento endodóntico de diente con necrosis pulpar y lesión periapical con instrumentos Reciproc Blue y XP-Endo Finisher: reporte de caso

\author{
Lidiane Mendes Louzada \\ ORCID: https://orcid.org/0000-0002-3480-8433 \\ Universidade Estadual de Campinas, Brasil \\ E-mail: lidiane.mlouzada@gmail.com \\ Esdras Gabriel Alves-Silva \\ ORCID: https://orcid.org/0000-0003-3223-8518 \\ Universidade Estadual de Campinas, Brasil \\ E-mail: esdras0702@yahoo.com.br \\ Beatriz Isabel Nogueira Lemos \\ ORCID: https://orcid.org/0000-0002-5230-7835 \\ Universidade Estadual de Campinas, Brasil \\ E-mail: lemos.bn@gmail.com \\ Marlos Barbos-Ribeiro \\ ORCID: https://orcid.org/0000-0003-4145-5565 \\ Universidade de Pernambuco, Brasil \\ E-mail: ribeiro.marlos@yahoo.com.br \\ Aline Vitória de Souza Nogueira \\ ORCID: https://orcid.org/0000-0002-9041-8966 \\ Universidade Estadual de Campinas, Brasil \\ E-mail: a193616@dac.unicamp.br \\ Brenda Paula Figueiredo de Almeida Gomes \\ ORCID: https://orcid.org/0000-0002-8449-0646 \\ Universidade Estadual de Campinas, Brasil \\ E-mail: bpfagomes@fop.br \\ Rodrigo Arruda-Vasconcelos \\ ORCID: https://orcid.org/0000-0002-0968-0212 \\ Universidade Estadual de Campinas, Brasil \\ E-mail: vasconcelosra@yahoo.com.br
}

\begin{abstract}
Resumo
O objetivo do tratamento endodôntico é prevenir ou eliminar a periodontite apical através da limpeza, modelagem e obturação do sistema de canais radiculares. O preparo químico-mecânico é a etapa do tratamento endodôntico com maior capacidade de promover a redução de microrganismos presente no interior dos canais radiculares. Com o objetivo de superar desafios anatômicos, especialmente casos com curvaturas, novos tratamentos térmicos às ligas dos instrumentos endodônticos têm sido propostos para aumento da flexibilidade. O objetivo do presente relato de caso clínico é descrever os procedimentos endodônticos realizados com Reciproc Blue e XP-Endo Finisher em um incisivo lateral superior com necrose pulpar e presença de lesão periapical. Paciente do sexo masculino foi encaminhado à clínica de Pós-Graduação da Faculdade de Odontologia de Piracicaba - UNICAMP com necessidade de tratamento endodôntico. O exame clínico relevou resposta negativa ao teste de sensibilidade pulpar ao frio. Radiograficamente foi observado presença de restauração na face mesial e presença de lesão periapical. $O$ tratamento endodôntico foi realizado sob magnificação através de microscópio operatório. Após anestesia local e abertura coronária, o tratamento endodôntico foi realizado utilizando instrumentos Reciproc Blue, com a técnica de desinfecção coroa-ápice. Foi realizada a patência e ampliação foraminal. O nível de instrumentação foi determinado em $1 \mathrm{~mm}$ além do forame apical. A substância química auxiliar empregada foi a clorexidina a $2 \%$ gel e solução salina estéril como solução irrigadora. Ao final do preparo químico-mecânico foi realizada a irrigação final com $3 \mathrm{~mL}$ de EDTA $17 \%$, sob agitação do instrumento XP-Endo Finisher por 1 minuto. Em seguida, o canal radicular foi obturado através da técnica de cone único utilizando guta-percha médium e cimento endodôntico (Endomethasone). O corte da guta-percha foi realizado com instrumento termoplastificador, $2 \mathrm{~mm}$ abaixo da junção amelo-cementária. O selamento coronário foi
\end{abstract}


realizado com a colocação de coltosol na entrada dos canais radiculares, seguido da inserção da resina composta fotopolimerizável pela técnica incremental.

Palavas-chave: Endodontia; Infecção endodôntica primária; Odontologia.

\begin{abstract}
The objective of the endodontic treatment is to prevento $r$ eliminate apical periodontitis by means of cleaning, shaping and filling the root canals. Chemomechanical preparation is recognized as one of the most important step for microbial reduction within the root canals. In order to overcome anatomical challenges, especially in cases of curvatures, novel heat treatments to endodontic instrument alloys have been proposed to increase its flexibility. The aim of the present clinical case report is to describe the endodontic procedures performed with Reciproc Blue and XPEndo Finisher files in an upper lateral incisor with pulp necrosis and presence of periapical lesion. Male patient was referred to the Postgraduate Clinic of the Piracicaba Dental School - UNICAMP seeking endodontic treatment. The clinical examination revealed negative response to the pulp sensitivity cold testing. Radiographically, the presence of restoration on the mesial surface and the presence of periapical lesion were observed. Endodontic treatment was undertaken under magnification by using an operating microscope. After local anesthesia and cavity access, endodontic treatment was performed using Reciproc Blue instruments, with the crown-down technique. Patency and foraminal enlargement were performed. The instrumentation level was determined at $1 \mathrm{~mm}$ beyond the apical foramen. $2 \%$ chlorhexidine gel was used as auxiliary chemical substance and sterile saline solution as an irrigating solution. At the end of the chemical-mechanical preparation, final rinse was performed with 3mL of 17\% EDTA, under agitation with the XP-Endo Finisher instrument for 1 minute. Then, the root canal was filled using the single cone technique using medium gutta-percha and endodontic cement (Endomethasone). The gutta-percha cut was performed with a thermoplasticizer instrument, $2 \mathrm{~mm}$ below the cementoenamel junction. Coronary sealing was performed by placing coltosol at the entrance of the root canals, followed by the insertion of light-curing composite resin using the incremental technique.
\end{abstract}

Keywords: Endodontics; Dentistry; Primary endodontic infection.

\title{
Resumen
}

El objetivo del tratamiento de endodoncia es prevenir la eliminación de la periodontitis apical mediante la limpieza, modelado y relleno de los conductos radiculares. La preparación quimiomecánica se reconoce como uno de los pasos más importantes para la reducción microbiana dentro de los conductos radiculares. Con el fin de superar los desafíos anatómicos, especialmente en casos de curvaturas, se han propuesto nuevos tratamientos térmicos para aleaciones de instrumentos de endodoncia para aumentar su flexibilidad. El objetivo del presente caso clínico es describir los procedimientos endodónticos realizados con limas Reciproc Blue y XP-Endo Finisher en un incisivo lateral superior con necrosis pulpar y presencia de lesión periapical. Un paciente de sexo masculino fue remitido a la Clínica de Posgrado de la Facultad de Odontología de Piracicaba - UNICAMP en busca de tratamiento endodóntico. El examen clínico reveló una respuesta negativa a la prueba de sensibilidad pulpar al frío. Radiográficamente se observó la presencia de restauración en la superficie mesial y la presencia de lesión periapical. El tratamiento de endodoncia se llevó a cabo con aumento mediante el uso de un microscopio quirúrgico. Después de la anestesia local y el acceso a la cavidad, se realizó el tratamiento endodóntico con instrumentos Reciproc Blue, con la técnica de corona hacia abajo. Se realizó permeabilidad y agrandamiento foraminal. El nivel de instrumentación se determinó a 1 mm más allá del agujero apical. Se utilizó gel de clorhexidina al $2 \%$ como sustancia química auxiliar y solución salina estéril como solución de irrigación. Al final de la preparación químico-mecánica, se realizó el enjuague final con 3 mL de EDTA al $17 \%$, bajo agitación con el instrumento XP-Endo Finisher durante 1 minuto. A continuación, se rellenó el conducto radicular mediante la técnica de cono único con gutapercha mediana y cemento endodóntico (endometasona). El corte de gutapercha se realizó con un instrumento termoplastificante, $2 \mathrm{~mm}$ por debajo de la unión cementoonamel. El sellado coronario se realizó colocando coltosol en la entrada de los conductos radiculares, seguido de la inserción de un composite fotopolimerizable mediante la técnica incremental.

Palabras clave: Endodoncia; Necrosis de la pulpa dental; Odontología.

\section{Introdução}

A periodontite apical é uma alteração inflamatória que afeta os tecidos periapicais e se caracteriza pela presença de infecções intrarradiculares, fatores de virulência microbiana (i.e., endotoxinas e ácido lipoteicóico) e liberação de biomarcadores inflamatórios, levando à reabsorção óssea (Marton \& Kiss 2014, Martinho et al. 2021).

O tratamento endodôntico tem como principal objetivo a prevenção ou eliminação da periodontite apical através da limpeza, modelagem e obturação do sistema de canais radiculares (Carvalho et al. 2019). O preparo químico-mecânico é a etapa do tratamento endodôntico com maior capacidade de promover a redução de microrganismos dos canais radiculares (Arruda-Vasconcelos et al. 2021, Barbosa-Ribeiro et al. 2019, 2021). Apesar dos avanços dos instrumentos endodônticos, 
nenhum deles é capaz de tocar todas as paredes dentinárias (Versiani et al. 2011, Neves et al. 2016, Carvalho et al. 2019), permitindo a manutenção de bactérias e restos de tecidos pulpares e debris.

Com o objetivo de superar desafios anatômicos, especialmente casos com curvaturas, novos tratamentos térmicos nas ligas metálicas dos instrumentos endodônticos têm sido propostos com o objetivo de aumentar a flexibilidade de tais instrumentos. Reciproc Blue é um sistema que utiliza o movimento reciprocante, ou seja, movimento alternado [( $\left.150^{\circ}\right)$ ação de corte no sentido anti-horário e ( $\left.30^{\circ}\right)$ liberação no sentido horário], desempenhando papel importante na instrumentação dos canais radiculares, uma vez que apresentam maior resistência à fadiga cíclica se comparados aos instrumentos rotatórios convencionais $\left(360^{\circ} \mathrm{em}\right.$ sentido horário). Além disso, apresenta tratamento termomecânico em sua superfície (De-Deus et al. 2017).

Os conceitos atuais em endodontia preconizam a agitação da solução irrigadora [i.e., clorexidina, hipoclorito de sódio, ácido etilenodiaminotetracético (EDTA)] para promover maior efetividade da substância e permitir maior toque às paredes dos canais radiculares e, consequentemente, maior redução da carga microbiana (Aveiro et al. 2021). A lima XP-Endo Finisher é um instrumento em NiTi, com diâmetro ISO 25 e sem conicidade (25/.00) que modifica sua forma, expandindo-se dentro do canal, pela ativação da temperatura corpórea. Foi introduzida como uma técnica suplementar para aumentar a efetividade da irrigação e desinfecção durante o preparo químico-mecânico (Carvalho et al. 2019). Assim, a lima XP-Endo Finisher tem sido indicada como coadjuvante ao tratamento endodôntico (Bao et al. 2017).

O objetivo do presente relato de caso clínico é descrever os procedimentos endodônticos realizados com Reciproc Blue e XP-Endo Finisher em um incisivo central superior com necrose pulpar e presença de lesão periapical.

\section{Metodologia}

O presente estudo foi aprovado pelo Comitê de Ética em Pesquisa em Seres Humanos da Faculdade de Odontologia de Piracicaba - UNICAMP, Piracicaba, SP, Brasil (protocolo número CAAE 86140218.0.0000.5418).

Paciente, sexo masculino, 38 anos de idade, leucoderma, compareceu à clínica de Pós-Graduação em Endodontia da Faculdade de Odontologia de Piracicaba - UNICAMP para tratamento endodôntico do dente 12.

Os exames clínicos e radiográficos revelaram pigmentação da restauração em resina composta. O teste de sensibilidade pulpar a frio (resposta negativa) foi realizado após isolamento relativo e secagem do dente com jato de ar. Inicialmente, foi realizado o teste com bolinha de algodão compatível ao tamanho do dente, embebida em EndoIce, a qual se manteve em contato com a superfície vestibular por, no máximo 5 segundos, no dente contralateral. Em seguida, foi realizado o teste no dente suspeito (como descrito). O teste elétrico (resposta negativa) foi primeiramente realizado no dente contralateral, sob isolamento relativo e após secagem do elemento dental. Para haver condução de corrente elétrica, a extremidade do eletrodo foi embebida em clorexidina gel. O teste de percussão vertical se revelou positivo e o teste de palpação negativo. Radiograficamente, foi observado coronariamente a presença de restauração na porção mesial e, apicalmente, a raiz apresentava curvatura para distal e presença de lesão periapical. O paciente não apresentou sintomatologia espontânea.

O paciente foi esclarecido quanto ao diagnóstico e plano de tratamento.

Todo o tratamento endodôntico foi realizado sob magnificação e iluminação provenientes de microscópio operatório (DF Vasconcellos S/A, São Paulo, SP, Brasil). A região do dente 12 foi anestesiada com Lidocaína 2\% com epinefrina 1:100.000 (DFL, Rio de Janeiro, Brasil) através de técnica infiltrativa vestibular e palatina.

Em seguida, foram utilizadas broca esférica diamantada \#1014 (KG Sorensen, São Paulo, SP, Brasil) para desgaste de esmalte e dentina. Após a penetração na câmara pulpar, foi utilizada broca diamantada esférica \#1016 (KG Sorensen, São Paulo, SP, Brasil), com movimentos suaves, com o objetivo de promover a forma de conveniência adequada de formato 
triangular com base voltada para incisal e vértice para o cíngulo.

Após a localização das entradas dos canais radiculares, realizada de forma visual e tátil (com sonda endodôntica de ponta reta), foi realizado isolamento absoluto com lençol de borracha (Madeitex, Indústria de artefatos de borracha inovatex ltda, São José dos Campos, SP, Brasil) e barreira gengival (Top Dam, FGM, Joinville, SC, Brasil) no dente envolvido, com o objetivo de evitar a aspiração ou deglutição de corpos estranhos, bem como prevenir infiltração por saliva (microrganismos).

Em seguida, o canal radicular foi preenchido com $1 \mathrm{~mL}$ clorexidina 2\% gel (Endogel, Essencial Pharma Ltd. Itapetininga, SP, Brasil) e foram utilizadas limas do tipo K de pequeno diâmetro (\#10 e \#15) para a exploração inicial do canal. O comprimento inicial preconizado foi o comprimento aparente do dente (CAD) $-4 \mathrm{~mm}$. A cada troca de lima, o canal radicular foi irrigado com $5 \mathrm{~mL}$ de solução salina estéril através de seringa e agulha $(27 \mathrm{G})$ e novamente preenchido com clorexidina. Após a exploração manual dos canais radiculares, o preparo químico-mecânico dos terços cervical e médio foi realizado com instrumentos endodônticos Reciproc Blue (Reciproc R25 e R40) (VDW, Munique, Alemanha) em movimento reciprocante, através de motor elétrico (VDW, Munique, Alemanha).

Em seguida, foi realizada a odontometria do canal radicular, com auxílio de localizador foraminal (VDW Gold Reciproc, Munique, Alemanha), e lima do tipo $\mathrm{K} \mathrm{n}^{\mathrm{o}} 25$ para obtenção dos comprimentos reais de cada um dos canais radiculares.

$\mathrm{O}$ instrumento endodôntico que mais se ajustou às paredes do canal radicular na região foraminal foi a lima do tipo K \#25 (Maillefer/Dentsply, Ballaigues, Suíça). Portanto, o diâmetro inicial do forame apical foi equivalente ao diâmetro de uma lima tipo $\mathrm{K} \# 25$, ou seja, lima anatômica inicial (LAI). A instrumentação do terço apical foi estabelecida em $1 \mathrm{~mm}$ além do forame apical, como preconizado pela disciplina de Endodontia da FOP-UNICAMP, com o objetivo de promover a desinfecção em toda a extensão do canal radicular. Além disso, foi realizada a ampliação do forame apical até o instrumento Reciproc Blue (R40) (VDW Gold Reciproc, Munique, Alemanha) (três diâmetros subsequentes à LAI, denominada lima anatômica final [LAF]).

Ao final do preparo químico-mecânico, o canal radicular foi irrigado com $10 \mathrm{~mL}$ de solução salina estéril e, em seguida, uma irrigação com $3 \mathrm{~mL}$ de EDTA 17\% foi realizada, sob agitação do instrumento XP-Endo Finisher (FKG Dentaire SA, La Chaux-de-Fonds, Suíça) acoplado ao motor elétrico (VDW, Munique, Alemanha), por 1 minuto, a $800 \mathrm{rpm}$ e $1 \mathrm{ncm}, 1$ $\mathrm{mm}$ aquém do comprimento real do dente, em movimentos de vai-e-vem com amplitude de $8 \mathrm{~mm}$ (Carvalho et al. 2019), para efetiva remoção da smear layer formada durante o preparo do canal radicular. Posteriormente, o canal radicular foi irrigado com $5 \mathrm{~mL}$ de solução salina para remoção do EDTA $17 \%$.

A secagem do canal radicular foi realizada com pontas de papel absorvente estéreis. A obturação do canal foi realizada em sessão única, com cones de guta-percha Medium (Konne Indústria e Comércio de Materiais Odontológicos Ltda., Belo Horizonte, $\mathrm{MG}$ ), calibrados em $0,55 \mathrm{~mm}$ (referente à uma lima $\mathrm{K} \mathrm{n}^{\circ} 55$, previamente desinfectados com clorexidina $2 \%$ gel) e cimemento endodôntico Endomethasone (Septodont, Saint-Maur-des-Fossés Cedex, França) pela técnica do cone único, posicionados a $2 \mathrm{~mm}$ aquém do forame apical. $\mathrm{O}$ corte dos cones de guta-percha foi realizado com instrumento termoplastificador (Termo Pack II, Easy Equipamentos Odontológicos, Belo Horizonte, MG, Brasil) 2 mm abaixo da junção amelo-cementária. A compactação do material obturador foi realizada através de compactação à frio com condensadores de tamanho compatível.

A restauração do dente foi realizada com a colocação de Coltosol (Vigodent, Coltene, Rio de Janeiro, RJ) na entrada dos canais radiculares, seguido de aplicação de ácido fosfórico a 37\% (Condac, FGM, Joinville, SC, Brasil) por 15 segundos em toda a cavidade, duas camadas de adesivo Single Bond (3M Dental Products, St. Paul, MN, EUA) e inserção de resina fotopolimerizável Z 350 (3M Dental Products, St. Paul, MN, EUA) pela técnica incremental.

$\mathrm{O}$ aspecto radiográfico após a realização do tratamento endodôntico evidencia um selamento adequado em toda a 
Research, Society and Development, v. 10, n. 13, e406101320823, 2021

(CC BY 4.0) | ISSN 2525-3409 | DOI: http://dx.doi.org/10.33448/rsd-v10i13.20823

extensão do canal radicular, além de selamento coronário adequado.

A Figura 1 mostra os aspectos radiográficos inicial e final.

Figura 1. Aspecto radiográfico: (A) inicial e (B) final.

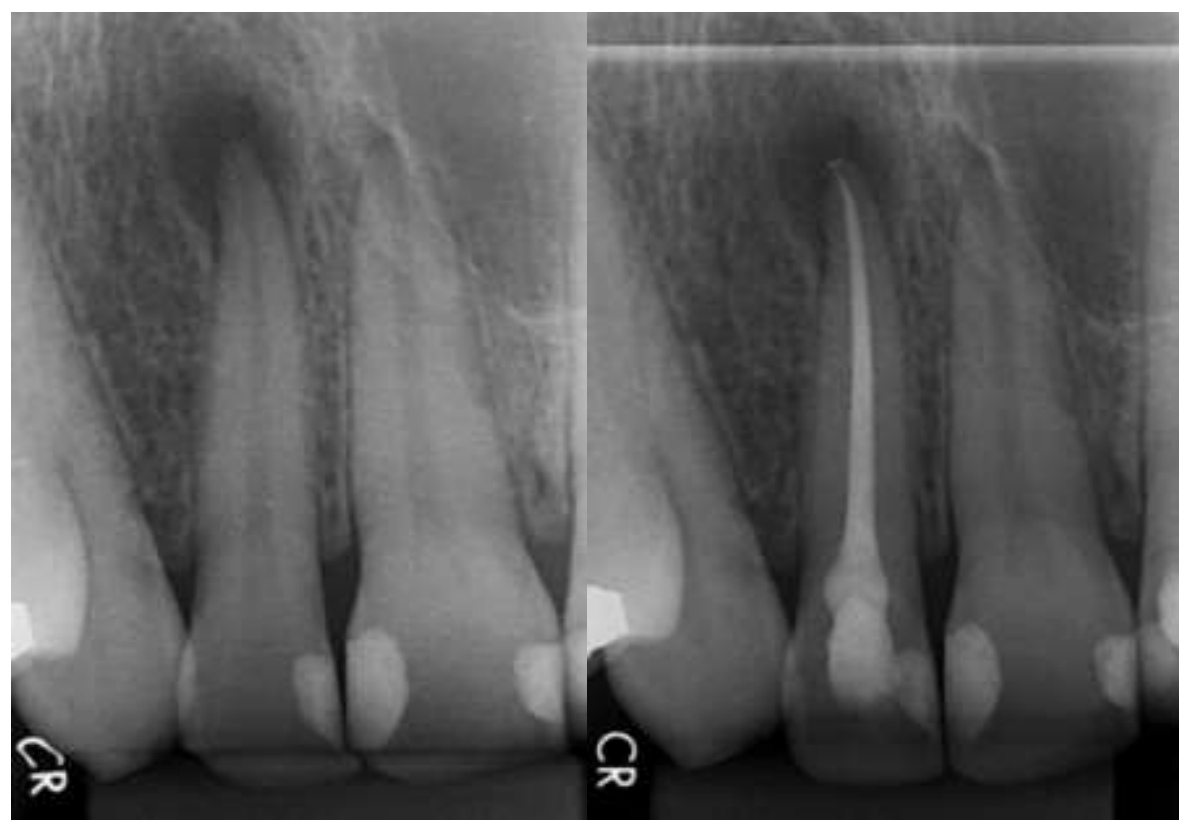

Fonte: Autores.

A Figura 2 mostra os aspectos clínicos dos procedimentos endodônticos. 
Figura 2. Aspectos clínicos: (A) Anestesia infiltrativa, (B) Abertura coronária, (C) Isolamento absoluto, (D) Fotopolimerização da barreira gengival, (E) Inundação do canal radicular com clorexidina 2\% gel, (F-G) Exploração do canal radicular com limas do tipo $\mathrm{K} \mathrm{n}^{\circ} 10$ e 15, (H) Instrumentação dos terços cervical e médio com instrumento Reciproc Blue R25, (I) Irrigação/aspiração com solução fisiológica estéril, (J) Odontometria eletrônica, (K) Instrumentação do terço apical com instrumento recicproc Blue R40, (L) Agitação do EDTA 17\% com XP-Endo Finisher, (M) Aspecto turvo do EDTA 17\% após agitação, (N) Secagem do canal radicular com cones de papel absorvente estéreis, (O-Q) Obturação do canal radicular com guta-percha e cimento Endomethasone e inserção de coltosol na entrada do canal radicular, (R) Restauração em resina composta, (S) Ajuste oclusal, (T) Aspecto clínico final.

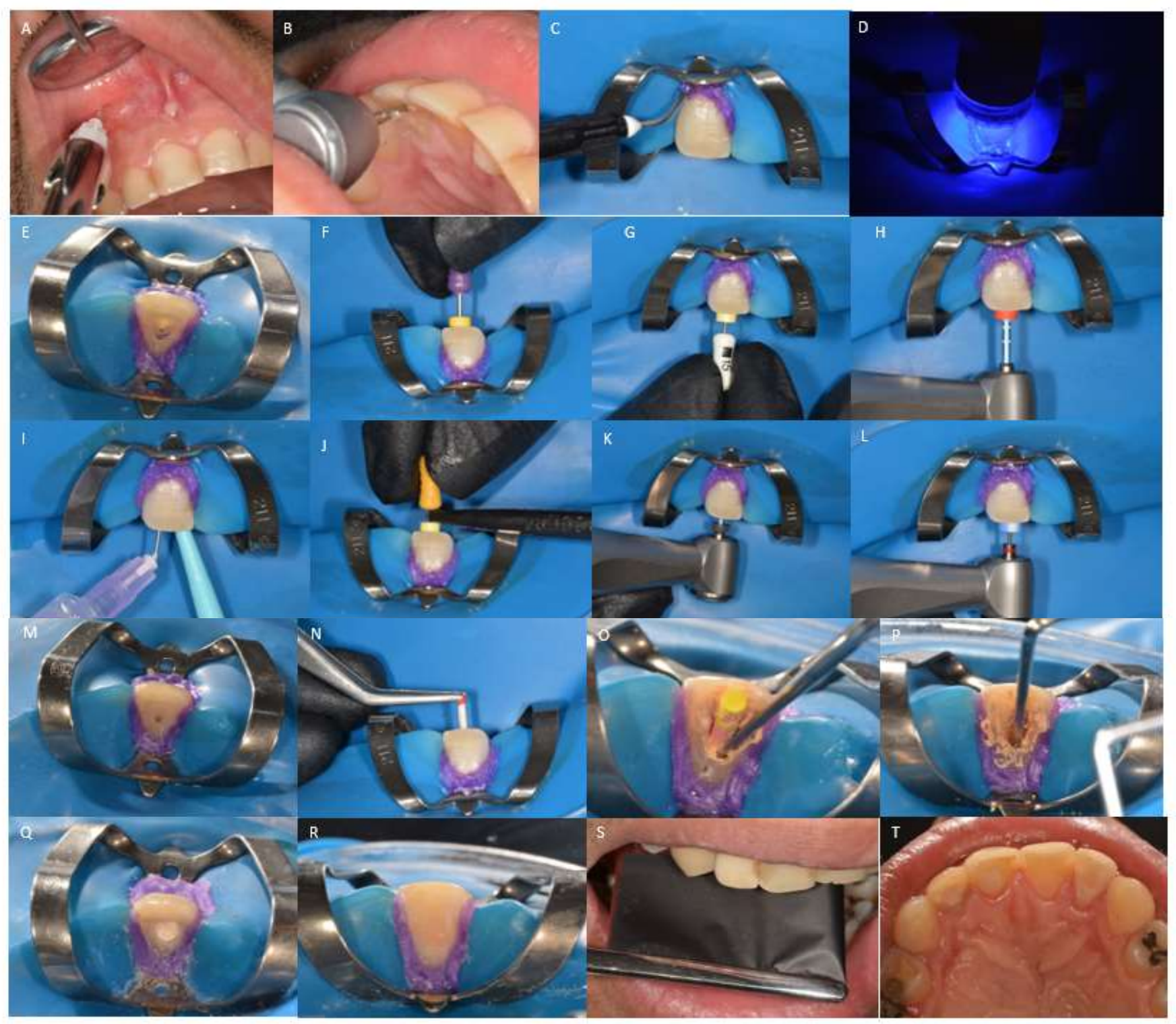

Fonte: Autores.

\section{Resultados e Discussão.}

Atualmente, diversos avanços tecnológicos têm sido incorporados aos procedimentos endodônticos, tais como utilização de radiografia digital, tomografia computadorizada de feixe cônico, desenvolvimento de novos 
sistemas/instrumentos, magnificação, laser, dentre outros. Com isso, a difusão de conhecimento, especialmente entre os clínicos e estudantes do curso de Odontologia foram fatores que motivaram a condução deste relato de caso clínico.

O conhecimento da anatomia dental é de fundamental importância para a condução de um tratamento endodôntico de excelência. Neste relato de caso, o tratamento endodôntico foi realizado em incisivo lateral superior. Este grupo dental normalmente possui comprimento médio de $22,1 \mathrm{~mm}$, raiz única, podendo apresentar 1 ou 2 canais radiculares em $97 \%$ e 3\%, respectivamente (De-Deus et al. 1992). Além disso, pode apresentar curvaturas acentuadas para a distal e lingual, podendo levar o clínico a cometer iatrogenias (desvios e/ou perfurações) na ausência de conhecimento da anatomia interna dental (Lopes \& Siqueira).

Atualmente, a realização do tratamento endodôntico no sentido coroa-ápice é recomendada. Esta técnica permite a descontaminação dos canais radiculares em diferentes terços (cervical, médio e apical), minimizando a possibilidade de extrusão apical de debris contaminados para além do forame apical e, consequentemente, reduzindo a possibilidade de o paciente apresentar dor pós-operatória ou flare-up (Barbosa-Ribeiro et al. 2018, Arruda-Vasconcelos et al. 2019).

A instrumentação com limas tratadas termicamente, como a Reciproc Blue, tem sido associada a maior flexibilidade do instrumento e maior resistência à fadiga (De-Deus et al. 2017). Com isso, é possível garantir maior segurança durante a instrumentação dos canais radiculares, além de respeitar a anatomia original do canal radicular em casos de curvaturas acentuadas, minimizando a formação de desvios apicais.

A substância química auxiliar empregada para a realização do tratamento endodôntico foi a clorexidina $2 \%$ gel. Tal substância é amplamente utilizada para o preparo químico-mecânico uma vez que apresenta amplo espectro microbiano, capacidade de lubrificação, ação reológica, tixotropia, substantividade e menor toxicidade quando comparada ao hipoclorito de sódio (Gomes et al. 2013, Barbosa-Ribeiro et al. 2018, Arruda-Vasconcelos et al. 2019). Diversos estudos mostraram a efetividade do preparo químico-mecânico realizado com clorexidina na redução da carga microbiana e de fatores de virulência em diferentes condições clínicas, incluindo infecções endodônticas primárias e secundárias, dentes com pulpite irreversível e lesão endodôntica-periodontal (Barbosa-Ribeiro et al. 2019, 2021, Louzada et al. 2020, Arruda-Vasconcelos et al. 2021).

A patência e limite de instrumentação (1 mm além do forame apical) foi realizado como preconizado na disciplina de Endodontia da FOP-UNICAMP. Estudos realizados mostraram que a ultrapassagem do forame apical, bem como a ampliação do mesmo permite a invaginação do tecido conjuntivo em direção ao canal radicular e formação de camada espessa de cemento na porção apical do canal radicular (selamento biológico) (Benatti et al. 1985). Além disso, os procedimentos de patência e ampliação foraminal não resultam em aumento de sintomatologia pós-operatória em tratamentos endodônticos realizados em sessão única, independente da condição pulpar (Sonoda 2011). Por fim, estas manobras clínicas asseguram maior desinfecção do sistema de canais radiculares, uma vez que garante a desinfecção em toda a extensão dos canais radiculares.

Apesar dos avanços tecnológicos, nenhum instrumento endodôntico é capaz de tocar todas as paredes dos canais radiculares (Versiani et al. 2013, Keleş et al. 2014). Com isso, cria-se um microambiente propício para a multiplicação bacteriana, podendo causar o insucesso do tratamento endodôntico. Assim, a agitação da substância química auxiliar se mostra importante na redução da carga microbiana no interior dos canais radiculares (Herrera et al. 2017). A utilização da XP-Endo Finisher se mostrou efetiva na redução da carga microbiana após a utilização da Reciproc Blue isoladamente (Carvalho et al. 2019). A XP-Endo Finisher apresenta diferentes comportamento dependendo das condições de temperatura a qual é submetida. Em temperatura ambiente, o instrumento é retilíneo (fase martensítica), ao passo que em temperatura corporal $\left(\sim 37^{\circ} \mathrm{C}\right) \mathrm{o}$ instrumento se altera e admite uma forma de "colher" com capacidade de expansão (fase austenítica), permitindo maior contato com as paredes dos canais radiculares (Teves et al. 2021).

O selamento coronário deve ser realizado com material que apresente excelente capacidade de adaptação marginal (i.e., resina composta) o mais breve possível. Isso se mostra importante, uma vez que a ausência de adaptação dos materiais 
restauradores temporários pode levar à micro infiltração marginal, permitindo a entrada de microrganismos presentes na saliva para o interior do sistema de canais radiculares, causando o insucesso do tratamento endodôntico (Ray \& Trope 1995). Além disso, tais materiais podem contribuir para a fragilidade do elemento dental, ocasionando fraturas.

\section{Considerações Finais}

O tratamento endodôntico de alta qualidade deve ser realizado em todos os casos clínicos. O profissional deve optar por empregar instrumentos e recursos tecnológicos com reconhecida efetividade comprovada cientificamente, além de se dedicar ao treinamento laboratorial previamente à realização de casos clínicos.

\section{Agradecimentos}

Este trabalho foi financiado pela Fundação de Amparo à pesquisa do Estado de São Paulo (FAPESP) (número de financiamento: 2015/23479-5, 2017/25242-8, 2019/10755-5, 2019/19300-0, 2019/09115-1, 2021/08372-0), CNPQ (número de financiamento: 308162/2014-5) e Coordenação de Aperfeiçoamento de Pessoal de Nível Superior Código Financiamento 001 da CAPES. Os autores negam quaisquer conflitos de interesse.

\section{Referências}

Arruda-Vasconcelos, R., Barbosa-Ribeiro, M., Louzada, L. M., Mantovani, G. D., \& Gomes, B. P. (2019). Apically Extruded Debris Using Passive Ultrasonic Irrigation Associated with Different Root Canal Irrigants. Braz Dent J. 30 (4):363-367.

Arruda-Vasconcelos, R., Louzada, L. M., Feres, M., Tomson, P. L., Cooper, P. R \& Gomes, B. P. F. A. (2021). Investigation of microbial profile, levels of endotoxin and lipoteichoic acid in teeth with symptomatic irreversible pulpitis: a clinical study. Int Endod J. 54 (1):46-60.

Aveiro, E., Chiarelli-Neto, V. M., de-Jesus-Soares, A., Zaia, A. A., Ferraz, C. C. R., Almeida, J. F. A., Marciano, M. A., Feres, M., \& Gomes, B. P. F. A. (2020). Efficacy of reciprocating and ultrasonic activation of $6 \%$ sodium hypochlorite in the reduction of microbial content and virulence factors in teeth with primary endodontic infection. Int Endod J. 53 (5):604-618.

Bao, P., Shen, Y., Lin, J., \& Haapasalo, M. (2017). In vitro efficacy of XP-endo Finisher with 2 different protocols on biofilm removal from apical root canals. J Endod. 43 (2):321-5.

Barbosa-Ribeiro, M., Arruda-Vasconcelos, R., de-Jesus-Soares, A., Zaia, A. A., Ferraz, C. C. R., Almeida, J. F. A \& Gomes, B. P. F. A. (2019). Effectiveness of calcium hydroxide-based intracanal medication on infectious/inflammatory contents in teeth with post-treatment apical periodontitis. Clin Oral Investig. 23 (6):2759-2766.

Barbosa-Ribeiro, M., Arruda-Vasconcelos, R., Fabretti, F. L., Silva, E. J. N. L., De-Deus G., \& Gomes, B. P. F. A. (2018). Evaluation of Apically Extruded Debris Using Positive and Negative Pressure Irrigation Systems in Association with Different Irrigants. Braz Dent J. 29 (2):184-188.

Barbosa-Ribeiro, M., Arruda-Vasconcelos, R., Louzada, L. M., Dos-Santos, D. G, Andreote, F. D., \& Gomes, B. P. F. A. (2021). Microbiological analysis of endodontically treated teeth with apical periodontitis before and after endodontic retreatment. Clin Oral Investig. 25 (4):2017-2027.

Benatti O., Valdrighi L., Biral R.R., Pupo J. (1985). A histological study of the effect of diameter enlargement of the apical portion of the root canal. J Endod. $11: 428-434$.

Carvalho, M. C., Zuolo, M. L., Arruda-Vasconcelos, R., Marinho, A. C. S., Louzada, L. M., Francisco, P. A., Pecorari, V. G. A., \& Gomes, B. P. F. A. (2019). Effectiveness of XP-Endo Finisher in the reduction of bacterial load in oval-shaped root canals. Braz Oral Res. 33 (1): 021.

De Deus, Q. D. Endodontia. (5a ed.), MEDSI, 1992.

De-Deus, G., Silva, E. J., Vieira, V. T., Belladonna, F. G., Elias, C. N., Plotino, G \& Grande, N. M. (2017). Blue Thermomechanical Treatment Optimizes Fatigue Resistance and Flexibility of the Reciproc Files. J Endod. 43 (3):462-466.

Gomes, B. P., Vianna, M. E., Zaia, A. A., Almeida, J. F \& Souza-Filho, F. J., Ferraz, C. C. (2013). Chlorhexidine in endodontics. Braz Dent J. 24 (2):89-102.

Herrera, D. R., Martinho, F. C., de-Jesus-Soares, A., Zaia, A. A., Ferraz, C. C. R., Almeida, J. F. A \& Gomes, B. P. F. A. (2017). Clinical efficacy of EDTA ultrasonic activation in the reduction of endotoxins and cultivable bacteria Int Endod J. 50 (10):933-940.

Kakehashi, S., Stanley, H. R \& Fitzgerald, R. J. (1965). The effects of surgical exposures of dental pulps in germ-free and conventional laboratory rats. Oral Surg Oral Med Oral Pathol. 20:340-9.

Keleş, A., Alcin, H., Kamalak, A \& Versiani, M. A. (2014). Oval-shaped canal retreatment with self-adjusting file: a micro-computed tomography study. Clin Oral Investig. 18 (4):1147-1153. 
Research, Society and Development, v. 10, n. 13, e406101320823, 2021

(CC BY 4.0) | ISSN 2525-3409 | DOI: http://dx.doi.org/10.33448/rsd-v10i13.20823

Lopes, H. P \& Siqueira Jr, F. J. Endodontia: Biologia e técnica. (4a ed.), Editora Elsevier. 2015.

Louzada, L. M., Arruda-Vasconcelos, R., Duque, T. M., Casarin, R. C. V., Feres, M \& Gomes, B. P. F. A. (2020). Clinical Investigation of Microbial Profile and Levels of Endotoxins and Lipoteichoic Acid at Different Phases of the Endodontic Treatment in Teeth with Vital Pulp and Associated Periodontal Disease. J Endod. 46 (6):736-747.

Martinho, F. C., Leite, F. R. M., Arruda-Vasconcelos, R., Louzada, L. M., Darveau, R. P \& Gomes, B. P. F. A. (2021) Influence of Bacterial Profiles in Cytokine and Clinical Features of Endodontic Disease. J Endod. 47 (8):1265-1271.

Marton, I. J \& Kiss, C. (2014). Overlapping protective and destructive regulatory pathways in apical periodontitis. J Endod. 40(1):155-163.

Neves, M. A. S., Provenzano, J. C., Roças, I. N \& Siqueira, J. F. (2016). Clinical antibacterial effectiveness of root canal preparation with reciprocating singleinstrument or continuously rotating multi-instrument systems. J Endod. 42 (1):25-9.

Ray, H. A \& Trope, M. (1995). Periapical status of endodontically treated teeth in relation to the technical quality of the root filling and the coronal restoration. Int Endod J. 28 (1):12-8.

Sonoda TN. (2011). Avaliação prospectiva da sintomatologia clínica pós-operatória de tratamentos endodônticos realizados em sessão única com patência e ampliação do forame apical. [Dissertação]. Campinas: Centro de Pesquisa São Leopoldo Mandic.

Teves, A., Blanco, D., Casaretto, M., Torres, J., Alvarado, D. E., Coaguila-Llerena, H., Faria, G \& Jaramillo, D. E. (2021). Multispecies biofilm removal by XP-endo Finisher and passive ultrasonic irrigation: A scanning electron microscopy study. Aust Endod J. doi: 10.1111/aej.12549. Epub ahead of print.

Versiani, M. A., Leoni, G. B., Steier, L., De-Deus, G., Tassani, S., Pécora, JD \& De Sousa-Neto, M. D. (2013). Micro-computed tomography study of ovalshaped canals prepared with the self-adjusting file, Reciproc, WaveOne, and ProTaper universal systems. J Endod. 39 (8):1060-6.

Versiani, M. A., Pécora, J. D \& De-Sousa-Neto, M. D. (2011). Flat-oval root canal preparation with self-adjusting file instrument: a micro-computed tomography study. J Endod. 37 (7):1002-7. 